\title{
Dealing with Social Isolation to Minimize Doctoral Attrition - A Four Stage Framework
}

\author{
Azad Ali \\ Indiana University of Pennsylvania \\ Indiana, PA - USA
}

azad.ali@iup.edu

\author{
Frederick Kohun \\ Robert Morris University, \\ Pittsburg, PA - USA \\ kohun@rmu.edu
}

\begin{abstract}
The purpose of this paper is to create a study model or framework for dealing with social isolation in doctoral programs. Previous studies have focused on the issue of attrition among doctoral students and the factors that cause doctoral students to drop out prior to completing their degrees. Among the factors that affect students' decisions to leave doctoral programs is the feeling of social isolation; however, the studies have focused on identifying the causes rather than establishing a framework for dealing with isolation feeling in doctoral programs. This paper intends to fill the gap and to establish a framework for dealing with isolation feelings in order to minimize doctoral attrition.
\end{abstract}

Keywords: Model for Doctoral Isolation, Isolation \& Doctoral Attrition, Social Isolation in doctoral programs.

Full version of this paper has been fast-tracked for publication in the International Journal of Doctoral Studies 